\title{
Phase I/II study of S-1 plus cisplatin combined with peptide vaccines for human vascular endothelial growth factor receptor 1 and 2 in patients with advanced gastric cancer
}

\author{
TORU MASUZAWA ${ }^{1}$, YOSHIYUKI FUJIWARA ${ }^{1}$, KAORU OKADA ${ }^{1}$, AYUMU NAKAMURA ${ }^{2}$, \\ SHUJI TAKIGUCHI ${ }^{1}$, KIYOKAZU NAKAJIMA ${ }^{1}$, HIROSHI MIYATA ${ }^{1}$, MAKOTO YAMASAKI ${ }^{1}$, \\ YUKINORI KUROKAWA ${ }^{1}$, RYUJI OSAWA ${ }^{3}$, KAZUYOSHI TAKEDA ${ }^{4}$, KOJI YOSHIDA ${ }^{3}$, \\ TAKUYA TSUNODA ${ }^{3}$, YUSUKE NAKAMURA ${ }^{3}$, MASAKI MORI $^{1}$ and YUICHIRO DOKI ${ }^{1}$

\footnotetext{
${ }^{1}$ Department of Gastroenterological Surgery, Graduate School of Medicine, Osaka University, Suita, Osaka;

${ }^{2}$ Department of Pharmacy, Osaka University Hospital, Suita, Osaka; ${ }^{3}$ Laboratory of Molecular

Medicine, Human Genome Center, Institute of Medical Science, The University of Tokyo, Tokyo;

${ }^{4}$ Department of Immunology, Juntendo University School of Medicine, Tokyo, Japan
}

Received March 24, 2012; Accepted May 22, 2012

DOI: $10.3892 /$ ijo.2012.1573

\begin{abstract}
The aim of this study was to evaluate the safety and efficacy of vaccination with human leukocyte antigen (HLA)-A24-restricted human vascular endothelial growth factor receptor 1 (VEGFR1)-1084 and VEGFR2-169 combined with chemotherapy in patients with advanced gastric cancer. HLA-A*2402-positive patients with advanced or recurrent adenocarcinoma of the stomach were vaccinated with VEGFR1-1084 and VEGFR2-169 combined with S-1 and cisplatin. The study included 22 patients (median age 60.5 years) who received at least one cycle of the combination therapy. No severe adverse effects caused by the vaccine therapy were observed except for an inflammatory reaction at the site of injection in 6 patients. Twelve patients (55\%) showed partial response and 10 had stable disease after two cycles of the combination therapy. The disease control rate (partial response and stable disease) was $100 \%$ after two cycles. The median time to progression was 9.6 months and median overall survival was 14.2 months. VEGFR1-1084specific cytotoxic T lymphocyte (CTL) response was induced in $18(82 \%)$ of the 22 patients and VEGFR2-169-specific CTL response was induced in $18(82 \%)$ of the 22 patients. Patients showing CTL response to VEGFR2-169 peptide had significantly better prognosis than those without, as demonstrated by the overall survival (OS) and time to progression (TTP) (OS, $\mathrm{p}=0.028$, TTP, $\mathrm{p}=0.006$ ). The combination therapy was well tolerated and highly effective in advanced or recurrent gastric cancer. Substantial specific CTL for both peptides was
\end{abstract}

Correspondence to: Dr Yoshiyuki Fujiwara, Department of Gastroenterological Surgery, Osaka University Graduate School of Medicine, 2-2 Yamada-Oka, Suita-City, Osaka 565-0871, Japan E-mail: yfujiwara@gesurg.med.osaka-u.ac.jp

Key words: peptide vaccine, VEGFR-1, VEGFR-2, gastric cancer, chemotherapy frequently induced even under chemotherapy. Thus, cancer vaccination combined with standard chemotherapy warrants further analysis as a promising strategy for the treatment of advanced cancer.

\section{Introduction}

Although the incidence of gastric cancer is declining worldwide, in 2008 gastric cancer remained the third leading cause of cancer-related mortality in men and the fifth in women (1). In Japan, gastric cancer is one of the most common causes of mortality, despite advances in diagnosis and treatment. In particular, unresectable or recurrent gastric cancer is associated with extremely poor prognosis even when treated with novel therapeutic agents, including taxanes [paclitaxel $(2,3)$ and docetaxel $(4,5)]$, irinotecan $(6,7)$, oxaliplatin $(8,9), S-1(10)$, and capecitabine (11), which are known to be efficacious in gastric cancer. A multi-center randomized controlled trial (SPIRITS trial) performed in Japan reported that the median overall survival (OS) and progression-free survival in patients with advanced gastric cancer treated with S-1 plus cisplatin were significantly longer in those treated with S-1 alone (10). Therefore, the Gastric Cancer Treatment Guidelines 2010 issued by the Japanese Gastric Cancer Association recommended an S-1 plus cisplatin combination regimen as a standard first-line treatment for unresectable and recurrent gastric cancer (12). However, even with this treatment, the median overall survival was 13 months and progression-free survival time was 6 months, suggesting the need for novel therapeutic modalities.

Immunotherapies, such as tumor antigen vaccination to induce antitumor $\mathrm{T}$ cells or antibodies, has been proposed as a novel treatment modality $(13,14)$. To date, a number of cancer-specific immunotherapies, particularly peptide vaccine therapies with cancer-testis antigens such as MAGE and NY-ESO-1, have been attempted $(15,16)$. Clinical trials have indicated that cancer vaccination with immunogenic epitope 
peptide derived from these antigens can induce specific $\mathrm{T}$ cell responses in cancer patients (17), however, the clinical response is considered to be limited due to possible immune evasion of tumor cells caused by downregulation or loss of human leukocyte antigen (HLA) and/or antigen proteins during tumor progression $(18,19)$.

Inhibition of angiogenesis is another promising strategy for cancer treatment $(20,21)$, and clinical trials have shown that administration of anti-vascular endothelial growth factor (VEGF) antibody combined with chemotherapy significantly prolonged the survival of colorectal cancer patients (22) and the progression-free survival in gastric cancer patients (23). Therefore, vaccine therapies targeting VEGF receptor 1 (VEGFR-1, also known as Flt-1) (24) and VEGFR-2 (25), both of which are overexpressed in endothelial cells of newly formed vessels in various types of primary and metastatic tumors $(26,27)$, are potentially effective anti-angiogenic cancer vaccines. In fact, we have already reported the efficacy of vaccine therapies with HLA-A*2402-restricted epitope peptides derived from VEGFR1 and VEGFR2 in a mouse model $(28,29)$.

In this study, we conducted a clinical trial to evaluate the safety and efficacy of vaccine therapy with VEGFR 1-1084 and/ or VEGFR2-169 combined with S-1 plus cisplatin in patients with unresectable or recurrent gastric cancer.

\section{Materials and methods}

Patient eligibility. Patients diagnosed with gastric adenocarcinoma considered unresectable or recurrent were enrolled in this trial at the Department of Gastroenterological Surgery, Osaka University Hospital (Japan). The following were the other main inclusion criteria: i) Eastern Cooperative Oncology (ECOG) performance status of 0 or 1; ii) age between 20 and 74 years; iii) adequate bone-marrow, cardiac, pulmonary, hepatic and renal functions including leukocyte count $2500-12000 / \mathrm{mm}^{3}$, neutrophil count $\geq 1500 / \mathrm{mm}^{3}$, platelet count $\geq 100,000 / \mathrm{mm}^{3}$, hemoglobin level $\geq 9.0 \mathrm{~g} / \mathrm{dl}$, aspartate aminotransferase and alanine aminotransferase $\leq 2.5 \mathrm{x}$ the institutional normal upper limits, total bilirubin $\leq 1.5 \mathrm{x}$ the institutional normal upper limits, creatinine equal or less the institutional normal upper limits, creatinine clearance using the Cockcroft-Gault formula $\geq 50 \mathrm{ml} / \mathrm{min}$; iv) life expectancy $>3$ months; v) no prior chemotherapy or one adjuvant regimen that did not include $\mathrm{S}-1$ or cisplatin and that was completed $>4$ weeks before entry to the study; vi) positive genomic DNA typing test for HLA-A*2402 (SRL, Tokyo, Japan); vii) signature of an informed consent. The main exclusion criteria were: i) the presence of another serious disease such as uncontrolled diabetes, hepatic disorder, cardiac disease, hemorrhage/bleeding; ii) pregnant or breastfeeding women; iii) patients who planned to become pregnant during the study period; iv) symptomatic infectious disease; v) concurrent treatment with steroids or immunosuppressive agents; vi) other uncontrolled malignant diseases; vii) unhealed wound; viii) intestinal obstruction or interstitial pneumonia; ix) decision of unsuitability by the principal investigator or the physician in charge.

Patient characteristics. Thirty patients were considered in this trial and 22 patients (73\%) were found to be HLA-A*2402 posi-
Table I. Clinicopathological data.

S1/CDDP+VEGFR vaccine

\begin{tabular}{lc}
\hline Number & 22 \\
Age (years) (mean $\pm \mathrm{SD})$ & $60.5 \pm 10.3$ \\
Sex & \\
$\mathrm{M}$ & 19 \\
$\mathrm{~F}$ & 3 \\
Disease progression & 16 \\
Unresectable & 6 \\
Recurrence & \\
Histological type & 7 \\
Differentiated & 15 \\
Undifferentiated & \\
HLA-A type & 11 \\
A*2402 homo & 11 \\
A*2402 hetero & \\
\hline
\end{tabular}

tive by DNA typing of HLA genomic variations and enrolled in this study between April 2008 and March 2010.

Table I shows the patient characteristics at study entry; patients included 19 males and 3 females. Sixteen patients had unresectable gastric cancer and 6 had recurrent disease after surgery. The tumor was considered unresectable for the following reasons: i) peritoneal dissemination and malignant ascites in 9 patients; ii) other distant organ metastasis in 4 patients; iii) distant nodal metastasis in 1 patient; and iv) more than one reason including i), ii) and iii) in 2 patients. Recurrent sites were as follows: i) peritoneal dissemination and malignant ascites in 3 patients; ii) liver or lung metastases with distal nodal metastasis in 2 patients; iii) local recurrence and pleural dissemination in 1 patient. Eleven patients had HLA-A*2402 homo type, and the other 11 patients had HLA-A*2402 and another HLA-A type such as $A^{*} 0201$. The patients received at least one cycle of combination therapy with chemotherapy plus peptide vaccination (1-18 cycles; median, 9 cycles).

Study design. This study was a non-randomized, open label, phase I and II clinical trial with VEGFR1-1084 and/or VEGFR2169 vaccines combined with standard chemotherapy, S-1 plus cisplatin, for advanced unresectable or recurrent gastric cancer. The primary endpoints were the safety of the combination therapy and the median time to disease progression (TTP). The secondary endpoints were immunological response, clinical response, accomplishment rate, 1 and 2 year survival rates, and the median survival time (MST). Toxicities were assessed by the Common Terminology Criteria for Adverse Events version 4.0 (CTCAE ver4.0). The dose-limiting toxicity was defined as a hematological toxicity of grade 4 and non-hematologic toxicity of grade 3 or greater. To assess the clinical response, computed tomography imaging was performed within a month before starting the first cycle and within 2 weeks after every two cycles. Every measurable region such as liver, lung or lymph node metastasis was evaluated by the Response Evaluation Criteria in Solid Tumors (RECIST) (30). Peptide-specific immunological responses were analyzed by IFN- $\gamma$ enzymelinked immunospot (ELISPOT) assay. Stage classification 
Table II. Complications observed during the two cycles of therapy.

$\mathrm{S}-1 / \mathrm{CDDP}+\mathrm{VEGFR}$ vaccine $(\mathrm{n}=22)$

\begin{tabular}{|c|c|c|c|c|c|}
\hline & G1 & G2 & G3 & G4 & G3-4 (\%) \\
\hline \multicolumn{6}{|l|}{ Blood/bone marrow } \\
\hline Leukopenia & 4 & 10 & 1 & 2 & $3(14)$ \\
\hline Neutropenia & 1 & 9 & 3 & 2 & $5(23)$ \\
\hline Lymphopenia & 1 & 8 & 4 & 1 & $5(23)$ \\
\hline Anemia & 5 & 13 & 4 & 0 & $4(18)$ \\
\hline \multirow[t]{2}{*}{ Thrombocytopenia } & 10 & 2 & 1 & 1 & $2(9)$ \\
\hline & G1 & G2 & G3 & G4 & $\mathrm{G} 2-4(\%)$ \\
\hline \multicolumn{6}{|l|}{ Hepatic } \\
\hline Increase in AST & 8 & 0 & 0 & 0 & $0(0)$ \\
\hline Increase in ALT & 7 & 0 & 0 & 0 & $0(0)$ \\
\hline Increase in bilirubin & 5 & 1 & 0 & 0 & $1(5)$ \\
\hline \multicolumn{6}{|l|}{ Renal } \\
\hline Increase in $\mathrm{Cr}$ & 6 & 0 & 0 & 0 & $0(0)$ \\
\hline Hyperkalemia & 9 & 0 & 0 & 0 & $0(0)$ \\
\hline \multicolumn{6}{|c|}{ Non hematological complications } \\
\hline Anorexia & 7 & 13 & 2 & 0 & $15(68)$ \\
\hline Cheilitis & 9 & 1 & 0 & 0 & $1(5)$ \\
\hline Vomiting & 8 & 1 & 0 & 0 & $1(5)$ \\
\hline Diarrhea & 10 & 2 & 0 & 0 & $2(9)$ \\
\hline Dysgeusia & 5 & 0 & 0 & 0 & $0(0)$ \\
\hline Fever & 2 & 3 & 0 & 0 & $3(14)$ \\
\hline Alopecia & 5 & 0 & 0 & 0 & $0(0)$ \\
\hline Flu-like symptoms & 2 & 1 & 0 & 0 & $1(5)$ \\
\hline Maculopapular rash & 4 & 0 & 0 & 0 & $0(0)$ \\
\hline Reaction at injection site & 4 & 0 & 2 & 0 & $2(9)$ \\
\hline
\end{tabular}

All adverse events were scored by CTCAE ver 4.0. AST, aspartate aminotransferase; ALT, alanine aminotransferase.

and the assessment of resected specimens were performed according to the 14th edition of the Japanese Classification of Gastric Cancer (12). This trial was approved by the Osaka University Ethics Committee, and registered at UMIN (http:// www.umin.ac.jp; Trial registration ID: UMIN000005007), and carried out in accordance with the Helsinki declaration on experimentation on human subjects.

Peptides. HLA-A*2402-restricted CMV peptide (QYDPV AALF), GMP-graded VEGFR1-1084 peptide (SYGVLLWEIF) (28), and GMP-graded VEGFR2-169 peptide (RFVPDGNRI) (29) were synthesized by the American Peptide Company (Sunnyvale, CA) according to a standard solid-phase synthesis method and purified by reversed-phase high-performance liquid chromatography (HPLC). The purity (>90\%) and the identity of the peptides were determined by analytical HPLC and mass spectrometry, respectively.

Treatment protocol. The S-1 plus cisplatin regimen was based on that previously reported in the multicenter phase III SPIRITS trial (10). S-1 was administered orally twice daily for the first 3 weeks of a 5-week cycle. The dose of S-1 administered each time was calculated according to the body surface area as follows: $<1.25 \mathrm{~m}^{2}, 40 \mathrm{mg} ; 1.25-1.5 \mathrm{~m}^{2}, 50 \mathrm{mg} ;>1.5 \mathrm{~m}^{2}, 60 \mathrm{mg} /$ day. Cisplatin was administered by intravenous infusion at $60 \mathrm{mg} / \mathrm{m}^{2}$ on Day 8 of each cycle. Furthermore, $1 \mathrm{mg}$ VEGFR1-1084 and VEGFR2-169 were emulsified together with $1 \mathrm{ml}$ of incomplete Freund's adjuvant (Montanide ISA-51 VG, SEPPIC, Paris) and injected subcutaneously at inguen from side to side every week 5 times. Patients with more than one cycle of this treatment were enrolled. Toxicities within 2 cycles, the clinical response within 2 cycles and peptide-specific immunological response within 6 cycles were evaluated. S-1 and cisplatin were repeatedly administered until disease progression was considered to have occurred. Administration of peptide vaccines was continued after discontinuation of the regimen of S-1 plus cisplatin after consultation with the patient.

Isolation and stock of peripheral blood mononuclear cells. Peripheral blood cells were obtained from patients at the end of every cycle of the treatment. Peripheral blood mononuclear cells (PBMCs) were isolated immediately by Ficoll-Paque Plus density gradient solution (GE Healthcare, 
Little Chalfont, UK), suspended in Cell Banker (Juji Field, Tokyo), and frozen and stored in liquid nitrogen.

Enzyme-linked immunospot (ELISPOT) assay. To assess the specific CTL response, ELISPOT assay was performed following in vitro expansion. Frozen PBMCs derived from the same patient were thawed at the same time, and their viability was confirmed to be $>90 \%$. PBMCs $\left(5 \times 10^{5} / \mathrm{ml}\right)$ were cultured with $10 \mu \mathrm{g} / \mathrm{ml}$ of the respective peptide and $100 \mathrm{IU} / \mathrm{ml}$ of IL-2 (Novartis, Emeryville, CA) at $37^{\circ} \mathrm{C}$. The peptide was added to the culture at Day 0 and 7 (final concentration $10 \mu \mathrm{g} / \mathrm{ml}$ ), and cells were harvested after 2 weeks. Following CD $4^{+}$cell depletion by Dynal CD4-positive isolation kit (Invitrogen, Carlsbad, CA), the cells were used as responder cells in the ELISPOT assay. IFN- $\gamma$ ELISPOT assay was performed using the Human IFN- $\gamma$ ELISpot PLUS kit (MabTech, Cincinnati, $\mathrm{OH})$ according to the instructions supplied by the manufacturer. Briefly, HLA-A*2402-positive B-lymphoblast TISI cells (IHWG Cell and Gene Bank, Seattle, WA) were incubated with $20 \mu \mathrm{g} / \mathrm{ml}$ of VEGFR1-1084 peptide or VEGFR2-169 peptide overnight, then the residual peptide in the media was washed out to prepare peptide-pulsed TISI cells as the stimulator cells. Prepared CD4- cells were cultured with peptide-pulsed TISI cells $\left(2 \times 10^{4}\right.$ cells/ well) at $1 / 1,1 / 2,1 / 4$, and $1 / 8$ mixture ratio of responder cells and stimulator cells (R/S ratio) on 96-well plate (Millipore, Bedford, MA) at $37^{\circ} \mathrm{C}$ overnight. Non-peptidepulsed TISI cells were used as negative control stimulator cells. All ELISPOT assays were performed in triplicate wells. The plates were analyzed by the automated ELISPOT reader, ImmunoSPOT S4 (Cellular Technology, Cleveland, $\mathrm{OH}$ ) and ImmunoSpot Professional Software version 5.0 (Cellular Technology). The number of peptide-specific spots was calculated by subtracting the spot number in the control well from the spot number of wells with peptide-pulsed TISI cells. The CTL response was considered positive when the average of peptide-specific spot number of three wells was $>15 /$ well and
Table III. Treatment response after the first two cycles.

S-1/CDDP+VEGFR vaccine

\begin{tabular}{lc}
\hline $\begin{array}{l}\text { Number } \\
\text { Achievement rate } \\
\text { of } 2 \text { cycle therapy }\end{array}$ & 22 \\
$\begin{array}{l}\text { Dose down of chemotherapy } \\
\text { Response evaluation }\end{array}$ & $10(45 \%)$ \\
PR & \\
SD & 12 \\
PD & 10 \\
Response rate & 0 \\
Disease control rate & $12(55 \%)$ \\
\hline
\end{tabular}

One patient decided to discontinue S-1+CDDP after one cycle for reasons unrelated to the side-effects.

the significant difference $(\mathrm{p}<0.05)$ was demonstrated between the average spot numbers. The sensitivity of our ELISPOT assay was periodically estimated as approximately average by the ELIPOT panel of Cancer Immunotherapy Consortium (CIC).

Statistical analysis. Statistical analysis was performed using the Student's t-test and Fisher's exact test. TTP and OS curves were estimated using the Kaplan-Meier methodology. All statistical analyses were performed with JMP 8.0.2 (SAS Institute, Cary, NC).

\section{Results}

Toxicity. Table II lists the adverse effects recorded during the first two cycles of the combination therapy. Grade 3 or 4 neutropenia and anemia were observed in approximately $20 \%$
A

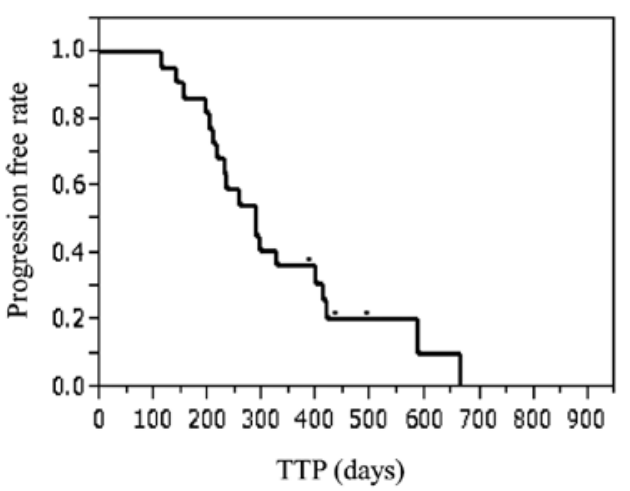

B

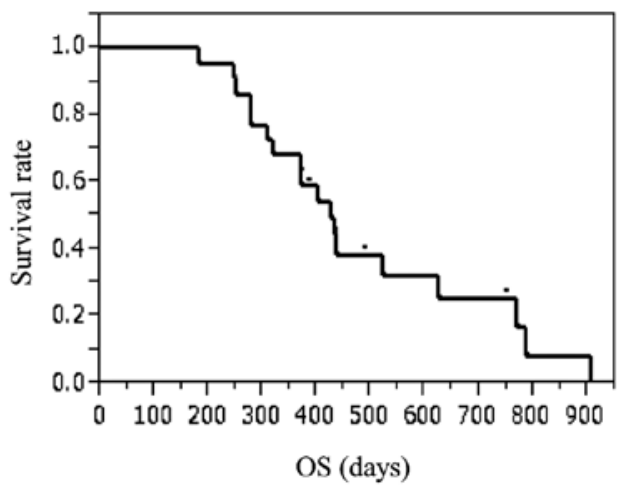

\begin{tabular}{ccccc}
\multicolumn{2}{c}{ MST months (days) } & OS & & \multicolumn{2}{c}{ Survival Rate } \\
TTP & & & 1 year & 2 years \\
9.6 months (288 days) & 14.2 months (427 days) & & $68.2 \%$ & $25.9 \%$ \\
\hline
\end{tabular}

Figure 1. Time to progression (TTP) and overall survival (OS) of all 22 patients. (A) TTP, the median TTP was 288 days (9.6 months); (B) OS, the median survival time (MST) was 427 days (14.2 months). 
A

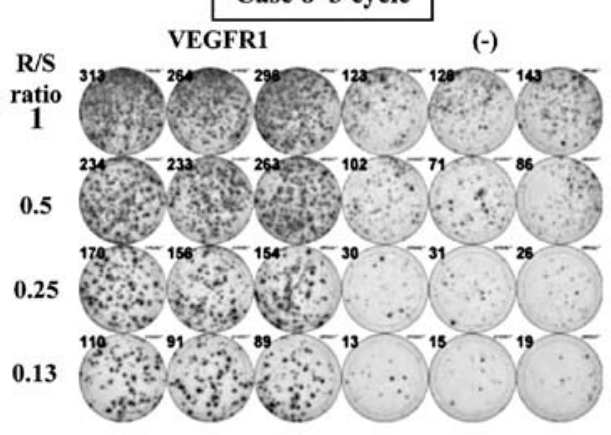

C

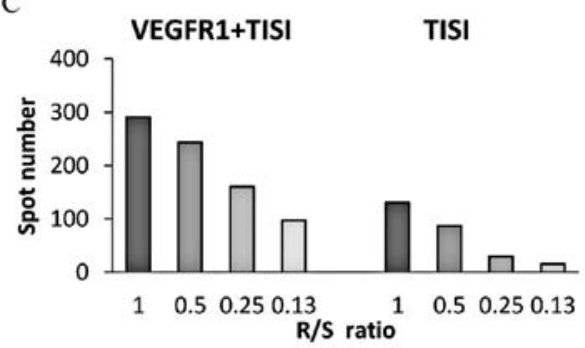

B

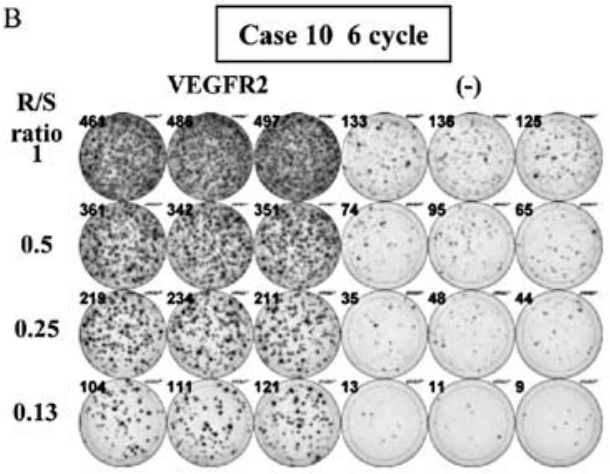

D

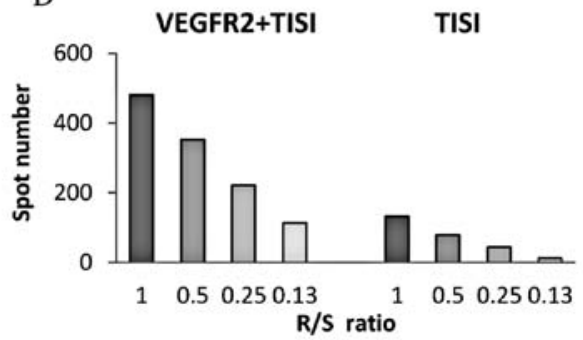

Figure 2. Immunological analysis. ELISPOT assay was performed to examine immunological response using peripheral blood mononuclear cells (PBMCs), which were collected after every treatment cycle. (A) Representative image of R1 specific spots in Patient 8; (B) representative image of R2 specific spots in Patient 10; (C,D) average number of specific spots in each $\mathrm{R} / \mathrm{S}$ ratio. $\mathrm{R} / \mathrm{S}$ ratio, responder/stimulator ratio.

A

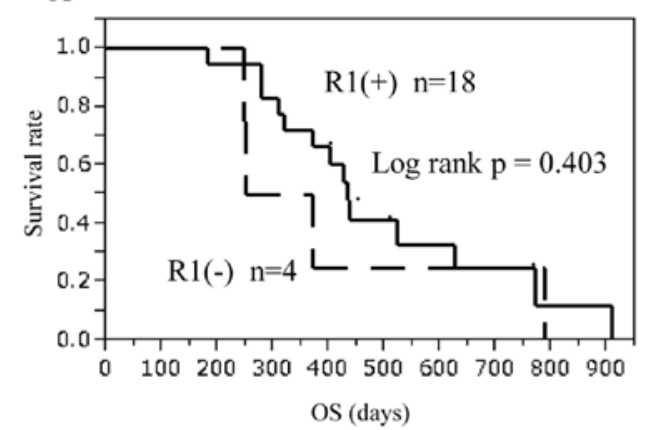

C

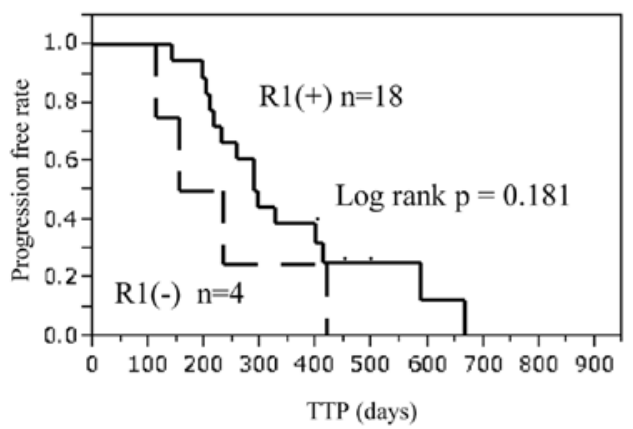

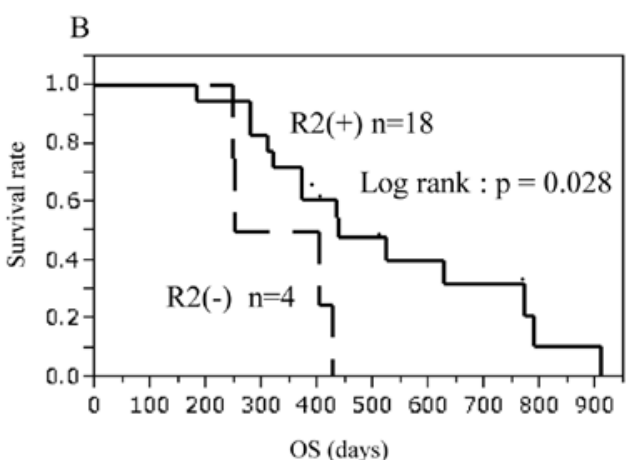

D

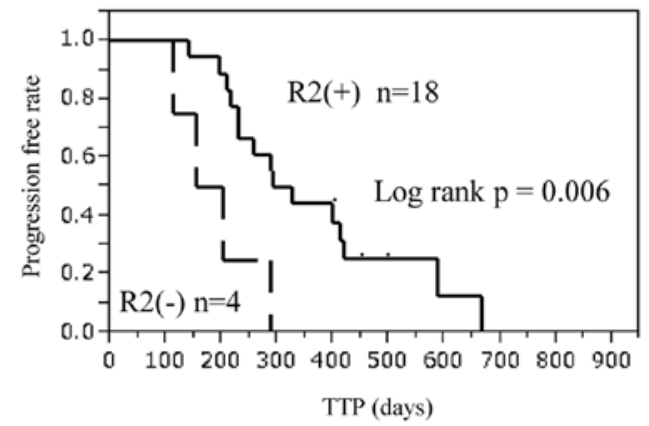

Figure 3. OS and TTP curves according to the results of CTL responses. (A) OS of 18 patients with positive (+) and 4 patients with negative (-) response to R1 peptide; (B) OS of R2 peptide; (C) TTP of R1 peptide; (D) TTP of R2 peptide.

of the patients. Anorexia of grade 2 or more was reported by $70 \%$ of the patients. During the vaccination therapy, 6 patients developed a reaction at the injection site and 2 patients developed an ulcer at the injection sites. No delayed wound healing or gastrointestinal bleeding was seen during the therapy. The dosage of chemotherapeutic agents, cisplatin and/or S-1, was reduced in 10 patients, however none of the patients dropped out of this study due to the above adverse effects. One patient discontinued chemotherapy with $\mathrm{S}-1$ plus cisplatin after 1 cycle but this was not related to any adverse effect. The same patient continued the peptide vaccination with the 2 nd line chemotherapy. 


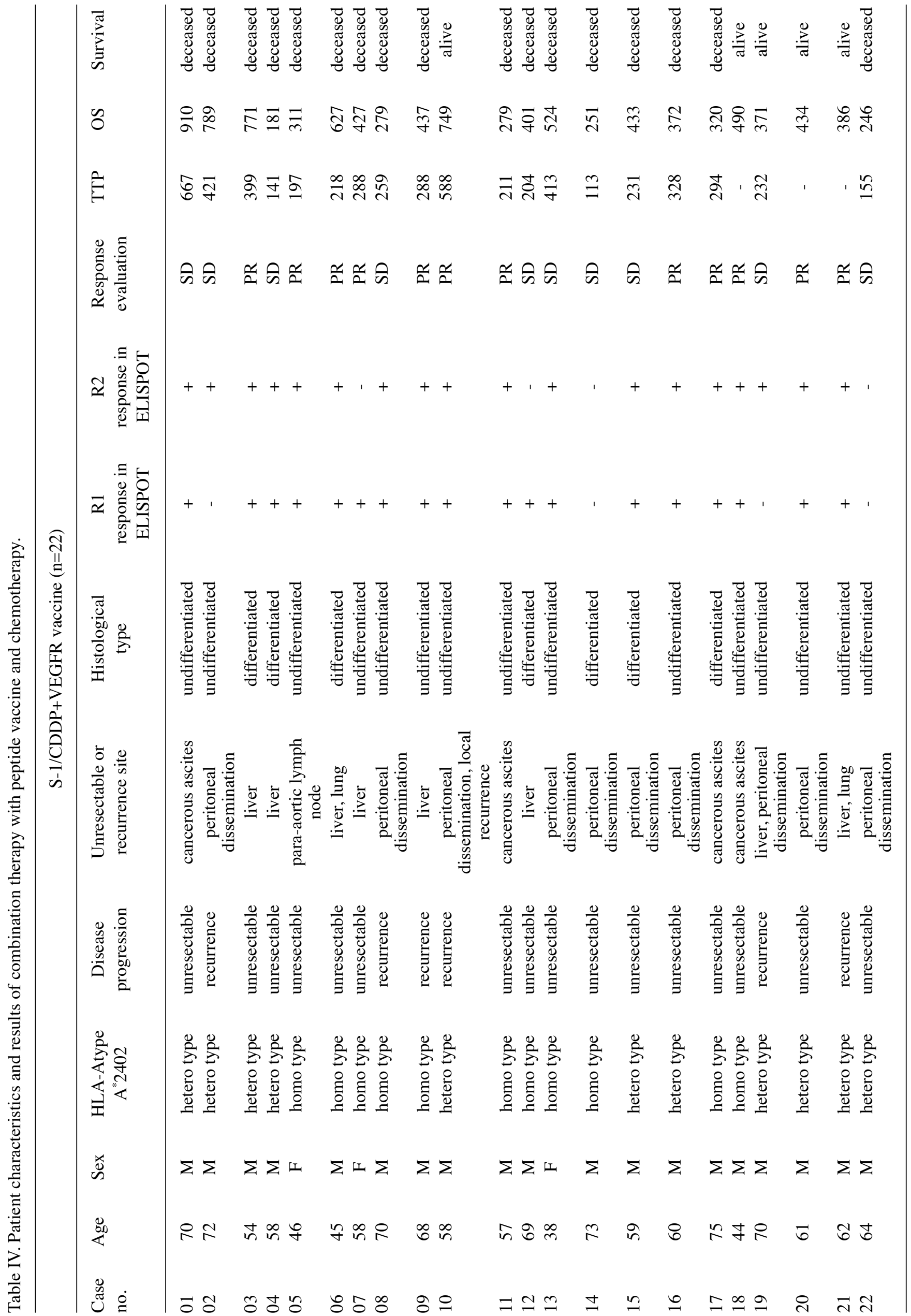


Clinical response and overall survival. Patients received a median of 9 cycles (range 1-18) of the combination therapy with S-1 plus cisplatin and peptide vaccination. Table III shows the clinical response date of the 22 patients. Twenty-one of the 22 patients received 2 or more cycles of the therapy. The clinical responses were classified as partial response (PR) in 12 patients $(54.5 \%)$; stable disease (SD) in 10 patients $(45.5 \%)$; and none of the patients showed progressive disease (PD). The response rate (RR) was $54.5 \%$ and disease control rate $(\mathrm{PR}+\mathrm{SD})$ was $100 \%$ after two cycles. One patient achieved disappearance of peritoneal dissemination and malignant ascites after 4 cycles of the treatment and underwent complete resection ( $\mathrm{R} 0$ resection).

Eighteen of the patients succumbed to the disease and none of the mortalities were due to other causes. The median TTP was 288 days (9.6 months), and MST was 427 days (14.2 months). Seven patients died within one year with a 1-year survival rate of $68.2 \%$. The other 8 patients died in the second year, with a 2-year survival rate of $25.9 \%$ (Fig. 1).

Immunological monitoring. The CTL response against VEGFR1-1084 after 3 cycles of the treatment in Patient 8 and the CTL response against VEGFR2-169 after 6 cycles of the treatment in Patient 10 are presented in Fig. 2 as representative results of positive immune responses. Eighteen (82\%) of the 22 patients who received at least one course of the vaccination showed positive CTL response against VEGFR1-1084, and 18 patients $(82 \%)$ showed positive CTL response against VEGFR2-169). Sixteen patients showed response to both peptides and only two patients showed no response to the vaccination.

Patients with a positive response to VEGFR2-169 peptide had significantly better prognosis compared with those with no response in OS $(p=0.028)$ and TTP $(p=0.006$, Fig. 3$)$. Patients with induced CTL response to VEGFR1-1084 peptide tended to show better prognosis, but this was not statistically significant.

Table IV shows all patient characteristics and results of combination therapy with peptide vaccine and chemotherapy.

\section{Discussion}

In this clinical trial, antiangiogenic vaccination therapy combined with standard chemotherapy with S-1 plus cisplatin was well tolerated without any major side-effects in patients with advanced or recurrent gastric cancer. Furthermore, the combination therapy achieved promising results for overall response rate $(\mathrm{PR}, 55 \%)$ and the disease control rate $(\mathrm{PR}+\mathrm{SD})$ was $100 \%$ after two cycles of the treatment, with MST of 14.2 months, and TTP of 9.6 months. Non-hematological toxicities were generally mild and none was greater than grade 3. Grade 3 toxicities were anorexia, observed in only $9 \%$, and skin reaction, in $9 \%$ of the patients. The incidence of grade 3 or 4 anorexia and nausea was also the most common in the group assigned to the S-1 plus cisplatin (30 and 11\%) in the SPIRITS trial (10). The frequencies of the observed grade 3 or 4 hematological side-effects were $23 \%$ for neutropenia, $18 \%$ for anemia, and $2 \%$ for thrombocytopenia, which were less than those in the group assigned to the $\mathrm{S}-1$ plus cisplatin in the SPIRITS trial $(40,26$, and 5\%, respectively) (2). The only specific side-effect caused by the vaccine treatment was reaction at the injection sites in 6 patients. Grade 3 skin ulceration was observed in 2 patients, although it did not cause discontinuation of the vaccine treatment. Therefore, this protocol is considered to be safe and well tolerable.

Both VEGFR1-1084-specific and VEGFR2-169-specific CTL responses were observed in as many as $82 \%$ of the patients even under the combination therapy with standard chemotherapy in patients with gastric cancer. This finding is consistent with the results of a previous study, which reported that administration of the standard dose of S-1 did not impede immunological responses to peptide vaccination in patients with gastrointestinal tract cancer (31). Furthermore, promising therapeutic effects as well as safety and tolerance in a clinical phase I trial of the combination of VEGFR2-169 and gemcitabine have been reported for patients with advanced pancreatic cancer (32). Gemcitabine, a chemotherapeutic agent specific for pancreatic cancer, is reported to reduce the quantity of myeloid-derived suppressor cells and possibly augmented CTL-mediated antitumor immune responses in vivo (33-35). Moreover, a randomized clinical trial of cancer vaccine (ALVAC-CEA/B7.1 vaccine) combined with fluorouracil, leucovorin, and irinotecan showed that vaccine-mediated immunity was not affected by chemotherapy (36). Thus, some chemotherapeutic reagents do not seem to inhibit, but rather augment, the antitumor immune responses when combined with immunotherapy, although suitable dose and treatment schedules should be carefully analyzed in further studies. In this study, patients treated with the vaccine and standard chemotherapy showed prolonged median TTP (9.6 months) and MST (14.2 months), compared with the results of the multicenter phase III trial (SPIRITS trial) in which the median progression-free survival (PFS) was 6 months and MST was 13 months (10). Previous clinical trials showed that vaccine therapy improved OS but not TTP $(32,37)$. The remarkable improvement of TTP in our study indicates the additional effectiveness of peptide vaccine therapy in combination with chemotherapy. Furthermore, patients with induced specific CTL against VEGFR2-169 peptide showed significantly better prognosis (OS and TTP) than those without such response, emphasizing the beneficial effects of immunotherapy with regard to patient prognosis.

In conclusion, VEGFR1-1084 and VEGFR2-169 vaccine therapy combined with standard chemotherapy is promising and warrants further clinical development of the strategy.

\section{References}

1. Jemal A, Bray F, Center MM, Ferlay J, Ward E and Forman D: Global cancer statistics. CA Cancer J Clin 61: 69-90, 2011.

2. Narahara H, Fujitani K, Takiuchi $\mathrm{H}$, et al: Phase II study of a combination of S-1 and paclitaxel in patients with unresectable or metastatic gastric cancer. Oncology 74: 37-41, 2008.

3. Iwase H, Shimada M, Tsuzuki T, et al: A phase II multi-center study of triple therapy with paclitaxel, S-1 and cisplatin in patients with advanced gastric cancer. Oncology 80: 76-83, 2011.

4. Sulkes A, Smyth J, Sessa C, et al: Docetaxel (Taxotere) in advanced gastric cancer: results of a phase II clinical trial. EORTC Early Clinical Trials Group. Br J Cancer 70: 380-383, 1994.

5. Van Cutsem E, Moiseyenko VM, Tjulandin S, et al: Phase III study of docetaxel and cisplatin plus fluorouracil compared with cisplatin and fluorouracil as first-line therapy for advanced gastric cancer: a report of the V325 Study Group. J Clin Oncol 24: 4991-4997, 2006. 
6. Boku N, Ohtsu A, Shimada Y, et al: Phase II study of a combination of irinotecan and cisplatin against metastatic gastric cancer. J Clin Oncol 17: 319-323, 1999.

7. Bang YJ, Van Cutsem E, Feyereislova A, et al: Trastuzumab in combination with chemotherapy versus chemotherapy alone for treatment of HER2-positive advanced gastric or gastro-oesophageal junction cancer (ToGA): a phase 3, open-label, randomised controlled trial. Lancet 376: 687-697, 2010.

8. Cunningham D, Starling N, Rao S, et al: Capecitabine and oxaliplatin for advanced esophagogastric cancer. N Engl J Med 358: $36-46,2008$

9. Amarantidis K, Xenidis N, Chelis L, et al: Docetaxel plus oxaliplatin in combination with capecitabine as first-line treatment for advanced gastric cancer. Oncology 80: 359-365, 2011.

10. Koizumi W, Narahara H, Hara T, et al: $\mathrm{S}-1$ plus cisplatin versus S-1 alone for first-line treatment of advanced gastric cancer (SPIRITS trial): a phase III trial. Lancet Oncol 9: 215-221, 2008.

11. Hong YS, Song SY, Lee SI, et al: A phase II trial of capecitabine in previously untreated patients with advanced and/or metastatic gastric cancer. Ann Oncol 15: 1344-1347, 2004.

12. Japanese Gastric Cancer Association: Japanese classification of gastric carcinoma: 3rd English edition. Gastric Cancer 14: 101-112, 2011.

13. Boon T, De Plaen E, Lurquin C, et al: Identification of tumour rejection antigens recognized by T lymphocytes. Cancer Surv 13 23-37, 1992.

14. Rosenberg SA, Yang JC and Restifo NP: Cancer immunotherapy: moving beyond current vaccines. Nat Med 10: 909-915, 2004.

15. Mackensen A, Herbst B, Chen JL, et al: Phase I study in melanoma patients of a vaccine with peptide-pulsed dendritic cells generated in vitro from CD34(+) hematopoietic progenitor cells. Int J Cancer 86: 385-392, 2000.

16. Bender A, Karbach J, Neumann A, et al: LUD 00-009: phase 1 study of intensive course immunization with NY-ESO-1 peptides in HLA-A2 positive patients with NY-ESO-1-expressing cancer. Cancer Immun 7: 16-23, 2007.

17. Nestle FO, Alijagic S, Gilliet M, et al: Vaccination of melanoma patients with peptide- or tumor lysate-pulsed dendritic cells. Nat Med 4: 328-332, 1998

18. Dunn GP, Bruce AT, Ikeda H, Old LJ and Schreiber RD: Cancer immunoediting: from immunosurveillance to tumor escape. Nat Immunol 3: 991-998, 2002.

19. Khong HT and Restifo NP: Natural selection of tumor variants in the generation of 'tumor escape' phenotypes. Nat Immunol 3: 999-1005, 2002

20. Fong TA, Shawver LK, Sun L, et al: SU5416 is a potent and selective inhibitor of the vascular endothelial growth factor receptor (Flk-1/KDR) that inhibits tyrosine kinase catalysis, tumor vascularization, and growth of multiple tumor types. Cancer Res 59: 99-106, 1999.

21. Gerber HP, Kowalski J, Sherman D, Eberhard DA and Ferrara N: Complete inhibition of rhabdomyosarcoma xenograft growth and neovascularization requires blockade of both tumor and host vascular endothelial growth factor. Cancer Res 60: 6253-6258, 2000.

22. Hurwitz H, Fehrenbacher L, Novotny W, et al: Bevacizumab plus irinotecan, fluorouracil, and leucovorin for metastatic colorectal cancer. N Engl J Med 350: 2335-2342, 2004.

23. Kang Y, Ohtsu A, Van Cutsem E, et al: AVAGAST: A randomized, double-blind, placebo-controlled, phase III study of first-line capecitabine and cisplatin plus bevacizumab or placebo in patients with advanced gastric cancer (AGC). ASCO Annual Meeting, 2010.
24. Shibuya M, Yamaguchi S, Yamane A, et al: Nucleotide sequence and expression of a novel human receptor-type tyrosine kinase gene (flt) closely related to the fms family. Oncogene 5: 519-524, 1990.

25. Matthews W, Jordan CT, Gavin M, Jenkins NA, Copeland NG and Lemischka IR: A receptor tyrosine kinase cDNA isolated from a population of enriched primitive hematopoietic cells and exhibiting close genetic linkage to c-kit. Proc Natl Acad Sci USA 88: 9026-9030, 1991.

26. Ferrara N, Gerber HP and LeCouter J: The biology of VEGF and its receptors. Nat Med 9: 669-676, 2003.

27. Plate KH, Breier G, Weich HA and Risau W: Vascular endothelial growth factor is a potential tumour angiogenesis factor in human gliomas in vivo. Nature 359: 845-848, 1992.

28. Wada S, Tsunoda T, Baba T, et al: Rationale for antiangiogenic cancer therapy with vaccination using epitope peptides derived from human vascular endothelial growth factor receptor 2 . Cancer Res 65: 4939-4946, 2005.

29. Ishizaki H, Tsunoda T, Wada S, Yamauchi M, Shibuya M and Tahara $\mathrm{H}$ : Inhibition of tumor growth with antiangiogenic cancer vaccine using epitope peptides derived from human vascular endothelial growth factor receptor 1 . Clin Cancer Res 12: 5841-5849, 2006

30. Therasse P, Arbuck SG, Eisenhauer EA, et al: New guidelines to evaluate the response to treatment in solid tumors. European Organization for Research and Treatment of Cancer, National Cancer Institute of the United States, National Cancer Institute of Canada. J Natl Cancer Inst 92: 205-216, 2000.

31. Sato Y, Fujiwara T, Mine T, et al: Immunological evaluation of personalized peptide vaccination in combination with a 5-fluorouracil derivative (TS-1) for advanced gastric or colorectal carcinoma patients. Cancer Sci 98: 1113-1119, 2007.

32. Miyazawa M, Ohsawa R, Tsunoda T, et al: Phase I clinical trial using peptide vaccine for human vascular endothelial growth factor receptor 2 in combination with gemcitabine for patients with advanced pancreatic cancer. Cancer Sci 101: 433-439, 2010.

33. Suzuki E, Kapoor V, Jassar AS, Kaiser LR and Albelda SM: Gemcitabine selectively eliminates splenic Gr-1+/CD11b+ myeloid suppressor cells in tumor-bearing animals and enhances antitumor immune activity. Clin Cancer Res 11: 6713-6721, 2005.

34. Dauer M, Herten J, Bauer C, et al: Chemosensitization of pancreatic carcinoma cells to enhance $\mathrm{T}$ cell-mediated cytotoxicity induced by tumor lysate-pulsed dendritic cells. J Immunother 28 : 332-342, 2005.

35. Correale P, Cusi MG, Del Vecchio MT, et al: Dendritic cellmediated cross-presentation of antigens derived from colon carcinoma cells exposed to a highly cytotoxic multidrug regimen with gemcitabine, oxaliplatin, 5-fluorouracil, and leucovorin, elicits a powerful human antigen-specific CTL response with antitumor activity in vitro. J Immunol 175: 820-828, 2005.

36. Kaufman HL, Lenz HJ, Marshall J, et al: Combination chemotherapy and ALVAC-CEA/B7.1 vaccine in patients with metastatic colorectal cancer. Clin Cancer Res 14: 4843-4849, 2008.

37. Kantoff PW, Higano CS, Shore ND, et al: Sipuleucel-T immunotherapy for castration-resistant prostate cancer. N Engl J Med 363: 411-422, 2010. 